\title{
How accurate is the urine dipstick test for diagnosing urinary tract infection?
}

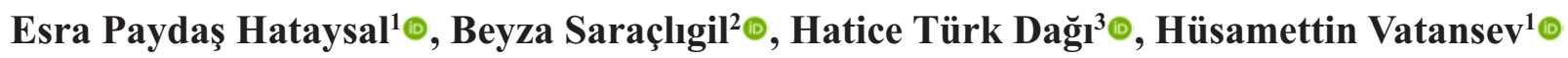 \\ ${ }^{1}$ Department of Biochemistry, Selçuk University School of Medicine, Konya, Turkey \\ ${ }^{2}$ Department of Biochemistry, Karatay University School of Medicine, Konya, Turkey \\ ${ }^{3}$ Department of Microbiology, Selçuk University School of Medicine, Konya, Turkey
}

DOI: $10.18621 /$ eurj.406215

\begin{abstract}
Objectives: Urine dipstick is the first step laboratory test to diagnose a urinary tract infection (UTI) which is a common infectious disease diagnosed in the laboratories. Early treatment of UTI is very important in order to prevent long-term complications. The gold standard to diagnose UTI is urine culture so there are a number of unnecessary urine culture requests. However, urine culture results are not available earlier than 24-36 hours. Besides, urine culture is expensive and causes time-consuming of treatment. Our aim in this study was to compare urine leukocyte esterase test and nitrite test of urine dipstick with urine culture to determinate the sensitivity, specificity, positive predictive value (PPV) and negative predictive value (NPV).

Methods: Urine culture results and urine dipstick test results of patients who admitted to Selçuk University Hospital between May 2016 and May 2017 were collected retrospectively. The study included 11.169 patients and 3299 of them have positive urine cultures.

Results: In total, 3299 (29.5\%) patients have positive urine culture. Out of these culture positive samples, positive dipstick results' ratios for leukocyte esterase and nitrite were $82.8 \%(\mathrm{n}=2733)$ and $22.4 \%(\mathrm{n}=774)$. Leukocyte esterase had 82.8\% sensitivity and 68.1\% specificity, with PPVs and NPVs of 52.1\% and 90.5\%, respectively.

Conclusions: The NPVs are found significantly higher than PPVs and it shows that urinary dipstick is more reliable to exclude disease than to diagnose the disease. Clinicians should not order urine culture from all patients without ant clinic signs.
\end{abstract}

Keywords: Urine culture, nitrite, leukocyte esterase, urine dipstick, urine analysis

Received: March 15, 2018; Accepted: June 22, 2018; Published Online: June 29, 2018

$\mathrm{U}$ rinary tract infection (UTI) is one of the most common infection diseases. Its' clinical symptoms vary from minimal dysuria to septic shock. UTI is the most common bacterial infection which diagnoses with the aid of clinical laboratories in young women and in the elderly [1].The second most common cause of bacteremia is UTI in hospitalized patients [2]. Many women experience UTI at least once in their whole lifetime [3].

The gold standard to diagnose UTI is urine culture so there are a number of unnecessary urine culture requests. However, urine culture results are not available earlier than 24 hours and this causes to delayed or inappropriate treatment [4]. Early treatment

Address for correspondence: Esra Paydaş Hataysal, MD., Selçuk University School of Medicine, Department of Biochemistry, Konya, Turkey E-mail: dr.esrapaydas@hotmail.com 
of UTI is important to prevent long-term complications. Additionally, urine culture test is expensive and causes delay of treatment [5]. Because of all these causes, a fast, easy, reliable, practicable and sensitive screening test is needed to begin treatment or rule out UTI as early as possible $[3,6]$.

Although the most common examination finding in patients with UTI is dysuria, dysuria is also the presenting complaint in another disease such as vaginitis. Patients with UTI sometimes show no typical symptoms and signs. Because of the fact that clinical symptoms or medical history are usually not enough to diagnose UTI, clinicians order lots of laboratory tests including urine culture, routine automated urinalysis even radiological tests to diagnose UTI. Nevertheless, suspected UTI is one of the most common indications for antimicrobial drugs use, with much prescribing of antimicrobials based on clinical symptoms and signs without confirmation by culture $[7,8]$.

Urine dipstick analysis is a quick, cheap and a useful test in predicting UTI. Because of the above mentioned limitations, urine analysis a preferred firststep investigation among clinicians.

The most frequently determined microorganism in urine culture is Escherichia coli in patients with UTI. Contamination is another frequently seen reason that causes positive culture results with a ratio of $29 \%-32 \%$ [3]. If the results of both nitrites and leukocyte esterase are negative, urine dipstick tests alone are useful in all populations to exclude the presence of infection rather than diagnose UTI in the presence of positive dipstick tests [9]. Several studies have examined the use of urinary dipstick tests to diagnose or rule out in UTI.

We aimed to compare urine leukocyte esterase test and nitrite test of urine dipstick with urine culture to determinate the sensitivity, specificity, positive predictive value (PPV) and negative predictive value (NPV).

\section{METHODS}

Dates are obtained between May, 2016 and May, 2017 from laboratory information systems of Selçuk University School of Medicine which is a central hospital with 934 beds. The study was approved by Selçuk University Defense research ethics committee.
Our electronic database (Enlil LIS) includes age, sex, main diagnosis, urine culture results and simultaneously automated urinalysis results. In the time period of our study, 15.487 requests of simultaneous urine cultures and urine dipstick tests. The dates of subjects who had simultaneously both urine dipstick test and urine culture test were evaluated in this study. All urine samples were completed within one hour after arrival to the laboratory in order to prevent contamination and overgrowth. If there is a patient with more than one request, only the first request evaluated.

For collection of the urine samples, sterile plastic urine sampling containers (Firat MedPlastik, Ankara, Turkey) were used. After the spot mid-stream urine samples arrived to laboratory, every specimen was analyzed within 1 hour.

For the urine dipstick chemical analysis, Dirui H800 (Dirui Industrial Co, Ltd, Zhengzhou China) was used. LE and Nitrite was measured with Dirui H11-800 strips(Dirui Industrial Co, Ltd, Zhengzhou China).

Nitrite in the urine is diazotized to form a diazonium compound and the diazonium compound reacting with tetrahydro-benzo-quinolin-3-phenol causes the color change. Granulocyte leukocytes in urine contain catalyze that catalyze the hydrolysis of pyrrole amino change acid ester to 3-hydroxy-5-pheny pyrrole. This pyrrole reacting with diazonium forms a purple colour.

LE results were reported as negative,trace, $1(+)$, $2(+)$ and $3(+)$. The nitrite results were reported as negative and positive. Cut-off values for a positive dipstick result was at least LE $(+)$ or nitrite $(+)$. Trace LE results were evaluated in a different category. For quality control, we made daily internal controls and 6 times in a year external quality control. (Randox Laboratories, Riqas, Ireland).

Urine cultures were performed in the Microbiology Laboratory of Selçuk University School of Medicine. Urine samples were inoculated in Columbia agar with 5\% sheep blood and eosin methylene blue agar (bioMerieux, France) by calibrated sterile loops. The plates were aerobically incubated $24-48$ hours at $35-37^{\circ} \mathrm{C}$. The colonies were then counted and $10^{5} \mathrm{cfu} / \mathrm{ml}$ was considered significant. Bacterial identification was performed with standard microbiological procedures and VITEK 


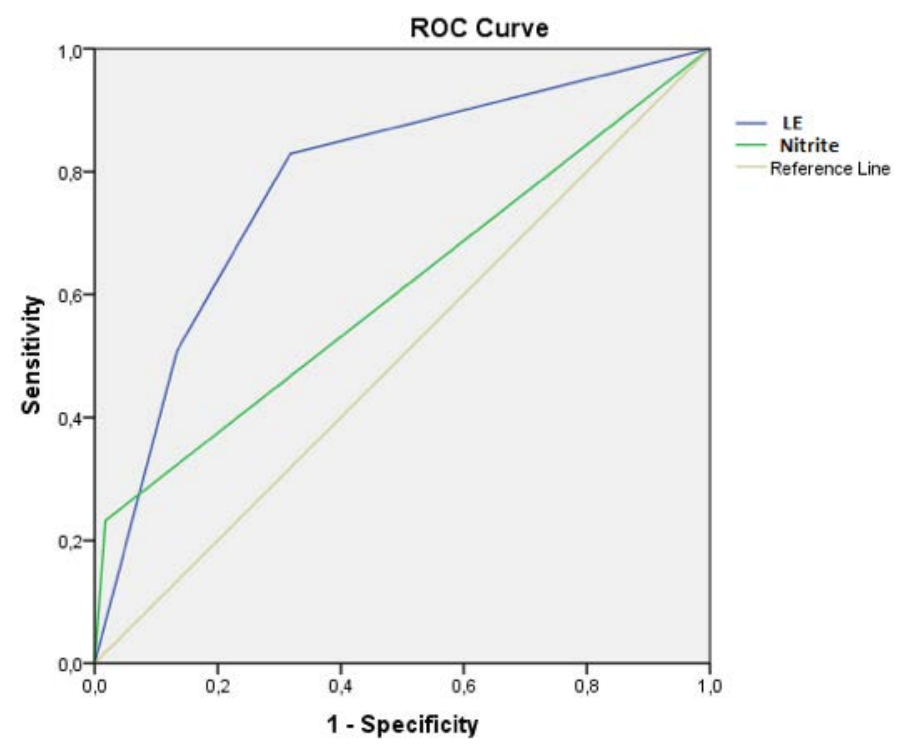

Figure 1. ROC curves for leukocyte esterase and nitrite. Area under curve (AUC) for Leukocyte Esterase $0.781(95 \% \mathrm{CI}=0.771$ $0.790)$ and $0.607(95 \% \mathrm{CI}=0.595-0.620)$ for Nitrite.

2 automated system (bioMerieux, France).

One thousand, four hundred and eighty-four subjects were excluded because of the fact that theirspecimens were accepted as contaminated. Two thousand, eight hundred and thirty-four subjects categorized differently because of trace LE results. In this retrospective and observational study, 11.169 individuals from 15.487 individuals remained.

\section{Statistical Analysis}

Statistical analysis performed by using SPSS version 15 (SPSS Inc, Chiacago, IL, USA). Calculation of sensitivity, specificity, positive and negative predictive values for LE and nitrite on dipstick was calculated as follows: Sensitivity $=$ True positive/(True positive + False negative), Specificity $=$ True negative/(True negative+False positive), Positive Predictive Value $=$ True positive $/($ True positive + False positive) and Negative Predictive Value $=$ True negative/(True negative + False negative) [5]. ROC analysis for LE and Nitrite on dipstick, Spearmen correlation between parameters were performed.

\section{RESULTS}

In our study, 11.169 subjects were evaluated [4,244 men (38\%), 6,925 women (62\%)] with the mean age of 25.9 years (ages ranges from 0 to 98 ).
Three thousand, two hundred and ninety-nine subjects (29.5\%) had positive urine cultures while 7870 specimens were negative. The predominant pathogen responsible for UTI was E. coli followed by Klebsiella spp. and Enterococcus spp. (Table 1).

In culture positive individuals, ratios of positive dipstick results for LE and nitrite were $82.8 \%(\mathrm{n}=$ $2733)$ and $23.4 \%(n=774)$. Leukocyte esterase had $82.8 \%$ sensitivity and $68.1 \%$ specificity, with PPVs and NPVs of $52.1 \%$ and $90.5 \%$, respectively. Nitrite had $22.4 \%$ sensitivity and $99.3 \%$ specificity, with PPVs and NPVs of $84.3 \%$ and $75.1 \%$, respectively. (Table 2). Nitrite had higher specificity and LE had higher sensitivity and NPV. Having trace LE subjects were evaluated, too. $82.4 \%$ of trace LE subjects were urine culture negative. The area under the curve (AUC) for LE was $0.781(95 \% \mathrm{CI}=0.771-0.790)$ and

Table 1. Identification of microorganisms from culture plates

\begin{tabular}{lc}
\hline Organisms & n (\%) \\
\hline E. coli & $1670(50.6 \%)$ \\
Klebsiella spp. & $405(12.2 \%)$ \\
Enterococcus spp. & $315(9.5 \%)$ \\
Candida spp. & $154(4.6 \%)$ \\
Proteus spp. & $132(4 \%)$ \\
Pseudomonas spp. & $120(3.6 \%)$ \\
\hline
\end{tabular}


Table 2. Performance characteristics of dipstick

\begin{tabular}{lccc}
\hline & LE & N & LE or N \\
\hline Sensitivity & $82.8 \%$ & $22.4 \%$ & $84.3 \%$ \\
Specificity & $68.1 \%$ & $99.3 \%$ & $66.7 \%$ \\
PPV & $52.1 \%$ & $84.3 \%$ & $51.4 \%$ \\
NPV & $90.5 \%$ & $75.1 \%$ & $91 \%$ \\
\hline
\end{tabular}

$\mathrm{LE}=$ leukocyte esterase test, $\mathrm{N}=$ Nitrate test, $\mathrm{PPV}=$

Positive predictive value, NPV $=$ Negative predictive value

for Nitrite AUC was $0.607(95 \% \mathrm{CI}=0.595-0.620)$ in ROC analysis which is shown in Figure 1. Urine culture accepted as a reference method for ROC analysis.

\section{DISCUSSION}

In our study, we checked the reliability of urine dipstick LE and nitrite test for diagnosing UTI. Our findings demonstrated that there are many unnecessary urine culture requests. Only $29.5 \%$ of patients were culture positive. Definite diagnosis of UTI is course urine culture but ordering urine culture from all patients without UTI symptoms causes increased labor force, payment and delay of results. Kayalp et al. [3], Okado et al. [10], Christenson et al . [11] and Öztürk et al. [12] reported higher unnecessary culture requests then ours with a percentage of $97.7 \%$ $, 80 \%, 82.1 \%$ and $88 \%$, respectively. As expected, E. coli was the most common isolated bacteria in urine samples. Our specificity and sensitivity findings were similar as literature. The nitrate test is less sensitive because it is affected by many factors such as nonnitrate-reducing bacteria and lack of dietary nitrate [13]. Because of such reasons, sensitivity of nitrite test was low in our study. Therefore, a negative urinary nitrate test result cannot rule out UTI. In a different study, Ercan et al. [14] found the sensitivity and specificity for LE $85 \%, 61 \%$ and for Nitrite $43.1 \%$ and 99\%, respectively. Öztürk et al. [12] found sensitivity and specificity for LE $76.4 \%, 64.2 \%$ and for Nitrite $40 \%$ and $99 \%$, respectively. Najeeb et al. [15] reported for LE $58.8 \%$ sensitivity, $75 \%$ specificity and for nitrite $51.4 \%$ sensitivity, $94 \%$ specificity considering culture as gold standard. Contrary to our study, they reported lower sensitivity for LE [15]. Memişoğulları et al. [5] reported $65 \%$ sensitivity and $64 \%$ specificity for LE. They also suggested evaluation both of urinary microscopic examination and dipstick test because of increase of reliability of urine analysis. Trace LE levels showed that most of trace samples have negative urine culture results. If there is no clinical UTI symptom, it can be evaluated as negative.

The limitation of our study is that because of being a retrospective study, we couldn't classify patients according to their symptoms.

\section{CONCLUSION}

The NPVs are found significantly higher than PPVs and urinary dipstick is more reliable to exclude disease than to diagnose the disease. Urinalysis can rightly rule out UTI in the majority of patients. However, our findings support that only urine dipstick analysis is not enough to diagnose UTI. Clinicians should make decisions according to clinical examination of the patients and order urine culture if the urine analysis results don't match with the patient's clinic. When the clinician would like to start empirical treatment without waiting for culture results, routine urine dipstick analysis might be reliable in preliminary exclusion of UTI.

\section{Authorship declaration}

All authors listed meet the authorship criteria according to the latest guidelines of the International Committee of Medical Journal Editors, and all authors are in agreement with the manuscript.

\section{Conflict of interest}

The authors disclosed no conflict of interest during the preparation or publication of this manuscript.

\section{Financing}

The authors disclosed that they did not receive any grant during conduction or writing of this study.

\section{REFERENCES}

[1] Patel HD, Livsey SA, Swann RA, Bukhari SS. Can urine dipstick testing for urinary tract infection at point of care reduce laboratory workload? J Clin Pathol 2005;58:951-4.

[2] Mambatta A, Jayarajan J, Rashme VL, Harini S, Menon S, 
Kuppusamy J. Reliability of dipstick assay in predicting urinary tract infection. J Family Med Prim Care 2015;4:265-8.

[3] Kayalp D, Dogan K, Ceylan G, Senes M, Yucel D. Can routine automated urinalysis reduce culture requests? Clin Biochem 2013;46:1285-9.

[4] Williams GJ, Macaskill P, Chan SF, Turner RM, Hodson E, Craig JC. Absolute and relative accuracy of rapid urine tests for urinary tract infection in children: a metaanalysis. Lancet Infect Dis 2010;10:240-50. [5] Memişoğulları R, Yüksel H, Ak Yıldırım H, Yavuz Ö. Performance characteristics of dipstick and microscopic urinalysis for diagnosis of urinary tract infection. Eur J Gen Med 2010;7:174-8.

[6] Whiting P, Westwood M, Watt I, Cooper J, Kleijnen J. Rapid tests and urine sampling techniques for the diagnosis of urinary tract infection (UTI) in children under five years: a systematic review. BMC Pediatr 2005;5:4.

[7] John AS, Boyd JC, Lowes AJ, Price CP. Clinical Chemistry/Use of dipstick testing to rule out UTI. Am J Clin Pathol 2006;126:428-36.

[8] Fahey T, Webb E, Montgomery AA, Heyderman RS. Clinical management of urinary tract infection in women: a prospective cohort study. Fam Pract 2003;20:1-6.

[9] Devillé W, Yzermans JC, van Duijn NP, Bezemer PD, wan der Windt $\mathrm{D}$, Bouter LM. The urine dipstick test useful to rule out infections. A meta-analysis of the accuracy. BMC Urol 2004;4:4.
[10] Okada H, Sakai Y, Miyazaki S, Arakawa S, Hamaguchi Y, Kamidono S. Detection of significant bacteriuria by automatedurinalysis using flow cytometry. J Clin Microbiol 2000;38:2870-2.

[11] Christenson RH, Tucker JA, Allen E. Results of dipstick tests, visual inspection, microscopic examination of urine sediment, and microbiological cultures of urine compared for simplifying urinalysis. Clin Chem 1985;31:448-51.

[12] Öztürk A, Gınıs Z, Hancı T, Yıldız Z, Taşlıpınar MY, Uçar F, et al. Evaluation of diagnostic performance of routine automated urinalysis and association between urinary tract infection and leukocytosis. Acta Medica 2015;4: 42-7.

[13] Mambatta AK, Jayarajan J, Rashme VL, Harini S, Menon S, Kuppusamy J. Reliability of dipstick assay in predicting urinary tract infection. J Family Med Prim Care 2015;4:265-8.

[14] Ercan Ş, Yücel N, Özer RS, Kaptanağası Orçun A. [Evaluation of diagnostic performances of nitrite and leukocyte esterase with respect to age and gender]. Türk Klinik Biyokimya Derg 2014;12:91-8. [Article in Turkish]

[15] Najeeb S, Munir T, Rehman S, Hafiz A, Gilani M, Latif M. Comparison of urine dipstick test with conventional urine culture in diagnosis of urinary tract infection. J Coll Physicians Surg Pak $2015 ; 25: 108-10$ 